\title{
Association of Malnutrition with Subsequent Malaria Parasitemia among Children Younger than Three years in Kenya: A Secondary Data Analysis of the Asembo Bay Cohort Study
}

\begin{abstract}
Catherine V. Donovan, ${ }^{1 \star}$ Peter McElroy, ${ }^{2}$ Linda Adair, ${ }^{1}$ Brian W. Pence, ${ }^{1}$ Aggrey James Oloo, ${ }^{3}$ Altaf Lal, ${ }^{4}$ Peter Bloland, ${ }^{5}$ Bernard Nahlen, ${ }^{6}$ Jonathan J. Juliano, ${ }^{7}$ and Steven Meshnick ${ }^{1} \dagger$

${ }^{1}$ Gillings School of Global Public Health, University of North Carolina, Chapel Hill, North Carolina; ${ }^{2}$ U.S. President's Malaria Initiative, Malaria Branch, U.S. Centers for Disease Control and Prevention, Atlanta, Georgia; ${ }^{3}$ Masinde Muliro University of Science and Technology, Kakamega, Kenya; ${ }^{4}$ Malaria Elimination Demonstration Project, Foundation for Disease Elimination and Control of India, Mandla, India; ${ }^{5}$ Global Immunization Division, U.S Centers for Disease Control and Prevention, Atlanta, Georgia; ${ }^{6}$ Eck Institute for Global Health, University of Notre Dame, Notre Dame, Indiana; ${ }^{7}$ Division of Infectious Diseases, School of Medicine, University of North Carolina, Chapel Hill, North Carolina

Abstract. Malaria and malnutrition remain primary causes of morbidity and mortality among children younger than 5 years in Africa. Studies investigating the association between malnutrition and subsequent malaria outcomes are inconsistent. We studied the effects of malnutrition on incidence and prevalence of malaria parasitemia in data from a cohort studied in the 1990s. Data came from the Asembo Bay cohort study, which collected malaria and health information on children from 1992 to 1996 in western Kenya. Infants were enrolled at birth and followed up until loss to follow-up, death, end of study, or 5 years old. Anthropometric measures and blood specimens were obtained monthly. Nutritional exposures included categorized Z-scores for height-for-age, weight-for-age, and weight-for-height. Febrile parasitemia and afebrile parasitemia were assessed with thick and thin blood films. Multiply imputed and weighted multinomial generalized estimating equation models estimated odds ratios $(\mathrm{OR})$ for the association between exposures and outcomes. The sample included 1,182 children aged 0-30 months who contributed 18,028 follow-up visits. There was no significant association between malnutrition and either incident febrile parasitemia or prevalent febrile parasitemia. Prevalence ORs for afebrile parasitemia increased from $1.07(95 \% \mathrm{Cl}: 0.89,1.29)$ to $1.35(1.03,1.76)$ as stunting severity increased from mild to severe, and from $1.16(1.02,1.33)$ to $1.35(1.09,1.66)$ as underweight increased from mild to moderate. Stunting and underweight did not show a significant association with subsequent febrile parasitemia infections, but they did show a modest association with subsequent afebrile parasitemia. Consideration should be given to testing malnourished children for malaria, even if they present without fever.
\end{abstract}

\section{INTRODUCTION}

Malaria and malnutrition remain primary causes of morbidity and mortality among children younger than 5 years. Recent estimates indicate 270,000 children younger than five globally died from malaria in $2018 .{ }^{1}$ In Africa, malaria remains a leading cause of childhood mortality. ${ }^{2}$ In 2019, 57.5 million African children (29\%) younger than 5 years were stunted (low heightfor-age [HAZ]), and 12.7 million (6.4\%) were wasted (low weight-for-height). ${ }^{3}$ East Africa and Kenya have particularly high burdens of malnutrition. More than one-third (36\%) of all children in East Africa are stunted. ${ }^{4}$ In Kenya alone, almost two million children (26\%) younger than 5 years were stunted in $2014 .^{4}$

Malnutrition, a syndrome of inadequate intake and absorption of protein, energy, and micronutrients combined with frequent infections that results in poor growth, ${ }^{5}$ affects a large proportion of children in malaria-endemic regions. Malnutrition may alter susceptibility to malaria infection, increase severity of the disease by weakening the immune system and preventing an adequate immune response against malaria, or influence therapeutic effectiveness of antimalarial drugs. ${ }^{6}$ Malnutrition can impair the adaptive and innate immune responses, both of which are involved in the immune response to malaria. ${ }^{7}$ The innate immune system limits parasite density, whereas the adaptive immune response works to remove the parasite. ${ }^{8}$ Malnutrition may impair children's ability to clear an infection, resulting in higher population prevalence or

\footnotetext{
*Address correspondence to Catherine V. Donovan, Gillings School of Global Public Health, University of North Carolina, 135 Dauer Dr., Chapel Hill, NC 27599. E-mail: cvdonovan@alumni.unc.edu
} †Deceased. increased severity of disease. Malnutrition may also increase children's susceptibility to infection, resulting in higher population incidence. Different types of malnutrition, such as stunting or wasting, may also have different effects on children's susceptibility and response to malaria.

Results of previous studies on the relationship between malnutrition and malaria have been inconsistent. ${ }^{9}$ Most previous studies investigated the association of malnutrition, measured as anthropometric Z-score, with clinical malaria incidence. Although most studies defined clinical malaria as fever with diagnostically confirmed malaria, at least one ${ }^{10}$ used a clinical definition only, without laboratory confirmation. Previous studies have used varying parasitemia thresholds depending on the local epidemiology of malaria. Two longitudinal studies found a significant association between malnutrition and increased malaria incidence, ${ }^{11,12}$ whereas two others found a significant association between wasting ${ }^{13}$ or stunting $^{14}$ and decreased malaria incidence. Most studies have found no statistically significant association between undernutrition and malaria. ${ }^{10,13,15-18}$ Inconsistency in the results of previous studies may be due to differences in study population; differences in study design aspects such as measures of malnutrition, malaria, or control for confounding; and other factors involved in the host-parasite relationship. ${ }^{19}$ Half of the previous studies were conducted in the era before widespread use of effective malaria interventions such as distribution of insecticide-treated nets (ITNs), indoor residual spraying, and treatment with artemisinin-based combination therapy. ${ }^{10,11,14-16}$

A proportion of malaria infections, especially in highly endemic areas, may be asymptomatic. ${ }^{20,21}$ Many asymptomatic infections go untreated, yet they can adversely affect children's health, contributing to low-grade inflammation, lower 
hemoglobin and platelet counts, ${ }^{21}$ chronic anemia, cognitive impairment, and reduced school performance. ${ }^{22}$ Asymptomatic infections can also contribute to ongoing malaria transmission. ${ }^{20,22}$ Only three previous studies investigated the association of malnutrition with asymptomatic parasitemia, also with inconsistent results. ${ }^{14,23,24}$

This study used a rich dataset from an era predating mass scale-up of malaria interventions to investigate whether incident or prevalent afebrile or febrile parasitemia was associated with prior malnutrition among children younger than 3 years in western Kenya. The recent slowing of progress in malaria control ${ }^{25}$ and continued increase in the absolute number of stunted children in Africa ${ }^{26}$ make elucidating the interactions between malaria and malnutrition a high priority. As the public health community strives to further reduce the burdens of both malnutrition and malaria, effective targeting of limited services and supplies will be crucial. Using data that were collected before the mass scale-up of malaria interventions allows for an analysis with greater power to detect associations, given the high prevalence of both malaria and malnutrition during this time period. The results may be used to prioritize interventions and research priorities as the global response for both malnutrition and malaria becomes more focused and strategic in an effort to meet long-term goals. ${ }^{27,28}$

\section{METHODS}

Study site and sample. Data for this study were collected from the Asembo Bay Cohort Study conducted in 15 villages in the Asembo Bay area of Siaya district in Nyanza Province, western Kenya, between 1992 and 1996. The study was designed to assess the epidemiology, entomology, immunology, host factors, molecular biology, antigenic variation, and population genetics of Plasmodium falciparum in a large cohort of women and children in an area with intense malaria transmission. Malaria was holoendemic in the study site, with an estimated entomologic inoculation rate of 0.75 infective bites per person per day. ${ }^{29}$ This area has two rainy seasons from March to May and October to December, and two dry seasons from January to February and June to September. During the study period, chloroquine was the only malaria intervention recommended by the Kenyan Ministry of Health for treatment of uncomplicated malaria. ${ }^{30}$ Bed nets were available but not systematically used.

Beginning in June 1992, all pregnant women in participating villages were identified during a monthly census by trained community health workers or birth attendants residing in the same village. Women who provided informed consent were enrolled in the study and visited every month at home by village monitors who administered standard questionnaires and obtained axillary temperatures, thick and thin blood smears, and capillary blood samples preceding delivery. Motherinfant pairs were visited within 2 weeks of birth, and then every 2 weeks thereafter. Siblings younger than 5 years were also enrolled and followed up with the same procedures. At each visit, questionnaires were administered and children's health was reported by the mother or an adult sibling or guardian. Axillary temperatures were obtained using a digital thermometer. Thick blood films were obtained for any children with an elevated axillary temperature $\left(\geq 37.5^{\circ} \mathrm{C}\right)$. Capillary blood, thick and thin blood films, and anthropometrics were also routinely collected every 4 weeks. Height and weight were measured in centimeters and kilograms (respectively) to the nearest 10th. Children who were old enough to stand were measured standing, and all others were measured recumbent. Blood films from children with an elevated axillary temperature $\left(\geq 37.5^{\circ} \mathrm{C}\right.$ ) were examined in the CDC/Kenya Medical Research Institute laboratory the day they were collected. Any participant with documented fever and malaria parasitemia was visited the following day and treated with a single dose of sulfadoxine/pyrimethamine (SP), as per the approved study protocol. Children with documented parasitemia and no fever were not treated; however, village monitors were available at any time between visits to take temperatures and blood smears for children who may have become ill. More severe illnesses were referred to local health facilities. Mother-infant pairs were followed up until loss to follow-up, death, end of the study, or the infant's fifth birthday. ${ }^{31}$

All blood films were stained with Giemsa, and parasites were visually identified. Hemoglobin concentration $(\mathrm{g} / \mathrm{dL})$ was measured using the HemoCue system (HemoCue, Anglholm, Sweden). ${ }^{32}$ Hemoglobin genotyping was performed retrospectively using a PCR method. ${ }^{33}$ Additional details regarding laboratory procedures and data collection have been described previously. ${ }^{31}$

Measures. Malnutrition. Z-scores comparing a child's length/HAZ, weight-for-age (WAZ), and weight-for-length/ height $(\mathrm{WHZ})$ to a reference population were calculated using the WHO Multi Centre Growth Reference Study. ${ }^{34}$ Linear growth was considered as length for children younger than 2 years, and height for children aged 2 years and older. For simplicity, we will refer to height as the linear growth measurement, regardless of the age of the child. Categorical exposure variables were used to allow for more flexibility in estimating the relationship between the exposures and the outcomes, which was found to depart from linearity on the log scale. Three different four-level ordinal categorical exposure variables were created for each $Z$-score measure: 1) HAZ: severe stunting $(\leq-3)$, moderate stunting $(>-3$ and $\leq-2)$, mild stunting ( $>-2$ and $<0$ ), and not stunted $(\geq 0)$; 2) WAZ: moderate underweight $(\leq-2)$, mild underweight $(>-2$ and $<0)$, not underweight $(\geq 0$ and $<1$ ), and overweight $(\geq 1)$; and 3$) \mathrm{WHZ}$ : moderate wasting $(\leq-2)$, mild wasting $(>-2$ and $<0)$, not wasted $(\geq 0$ and $<2$ ), and overweight $(\geq 2)$. Severe underweight and wasting were not included as exposure categories because the number of children in these categories was too small for analysis. If more than two height and weight measurements were made within a 1-month period, the Z-scores for these measurements were averaged together before creating categorical variables.

$Z$-scores outside plausible bounds were flagged and changed to missing, according to the following guidelines defined by the $\mathrm{WHO}^{34}$ : WAZ Z-score less than -6 or greater than 5; HAZ Z-score less than -6 or greater than 6 ; weightfor-height $Z$-score less than -5 or greater than 5 . Accordingly, 215 (0.5\%) HAZ, 54 (0.1\%) WAZ, and 223 (0.5\%) WHZ measurements were flagged and changed to missing.

Beyond the WHO guidelines for plausible Z-score records, there is a lack of research on methods to further assess the accuracy of longitudinal anthropometric data. A method to visually assess plausibility of height measurements was defined based on previously described methods. ${ }^{35}$ Height for each child was graphed over time and flagged for visual inspection if it indicated a decrease from the previous measure 
of more than $2 \mathrm{~cm}$ (regardless of the length of time between the two consecutive measures). Flagged heights that appeared as a "divot," deviating from the height trend, were discarded if the following point returned to the original height trend. The previous height was discarded if it appeared as a "peak," where it deviated above the height trend, and the following height returned to the original trend. The last height measurement on a child was not flagged for missing unless it was clear there was a loss of height from a consistent height trend in the previous measurements. If no immediate trend could be seen in the measurements before or after the flagged height, the flagged measurement remained in the data. In the original dataset, 1,057 (6\%) height measurements were flagged and visually inspected, and $847(<5 \%)$ were discarded. A similar method was not used to assess weight measures as a decrease, and subsequent rebound in weight is plausible, especially in young children.

Malaria. Both prevalent and incident parasitemia were investigated as outcomes. All malaria parasite infections were counted as prevalent cases, whereas infections were only considered incident cases if the previous month showed no parasites. Malaria infections were further categorized into afebrile and febrile parasitemia. We considered malaria infections to be afebrile if the child did not present with fever. A repeated measure, three-level, ordinal categorical variable was created indicating 1) no parasitemia, 2) afebrile parasitemia (presence of parasites without fever), and 3) febrile parasitemia (presence of parasites with fever-axillary temperature $\geq 37.5^{\circ} \mathrm{C}$ ). For analysis of prevalent parasitemia, the entire sample was included, and both afebrile and febrile parasitemia were defined as the presence of parasites with (febrile) or without (afebrile) fever in the month following the exposure, regardless of the child's malaria status when the exposure was measured. For incident parasitemia, the sample was restricted to visits with a negative blood film in the month before the outcome measurement, limiting the population to children who could be at risk for an incident infection when the outcome was measured. Incident afebrile parasitemia and incident febrile parasitemia were then defined as the presence of parasites with (febrile) or without (afebrile) fever in the month following exposure, among children with a negative blood film in the month before the outcome measurement (see Figure 1). Outcomes measured 2 and 3 months after the exposure were also considered. According to the study protocol, only children presenting with febrile parasitemia were treated. Therefore, repeated, prevalent, febrile infections may have been recrudescent infections posttreatment, whereas repeated, prevalent, afebrile infections likely resulted from untreated, persistent infections.

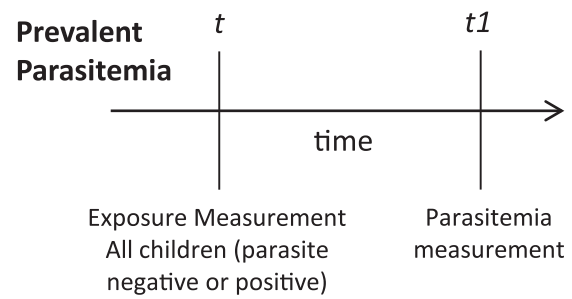

Covariates. Covariates were identified from a directed acyclic graph ${ }^{36}$ (DAG) informed by the literature (see Figure 2 ). Time-varying covariates included non-malarial anemia, ${ }^{37-39}$ season, ${ }^{40-42}$ age, ${ }^{40}$ and non-malarial illness. ${ }^{43-45}$ Non-malarial anemia was defined as a dichotomous variable indicating any anemia (<110 g/L hemoglobin) occurring without a concurrent parasite density (density $=0$ ), and not within 1 month following a febrile parasitemia infection. This was assessed in the month before the exposure. This variable was included as a proxy for iron-deficiency anemia, which may be a confounder of the relationship between malnutrition and malaria. However, given the numerous causes of anemia in this population, the effectiveness of this variable as a proxy for iron-deficiency anemia may be limited. Season was a three level-indicator variable denoting dry, rainy, and transition months. Non-malarial illness was a dichotomous variable assessed in the month before the exposure, indicating inability to drink, experience of chills, diarrhea, vomiting, difficulty breathing, or fever, in the absence of malaria parasitemia. Time was included as age (months from birth) and modeled as a cubic term, based on the quasi-Akaike information criterion comparing multiple functional forms. ${ }^{46}$

Time-fixed covariates included child gender, ${ }^{47}$ mother's education, ${ }^{48,49}$ socioeconomic status (SES), ${ }^{50,51}$ residence sector, ${ }^{50,52-54}$ maternal peripheral parasitemia at delivery, ${ }^{55,56}$ and sickle cell genotype. ${ }^{57,58}$ Mother's education was a dichotomous variable indicating less than 7 years of schooling or 7 years or more. Socioeconomic status was a dichotomous variable based on three indicators: ownership of more than one building within a compound, ownership of a bicycle, and ownership of a pressure lamp, based on perceptions of residents in the study area at the time of data collection. ${ }^{31}$ Villages were grouped into four different sectors and coded as indicator variables. Maternal peripheral parasitemia density was a dichotomous variable indicating the presence or absence of peripheral blood parasitemia at delivery. Sickle cell genotype was coded as indicator variables (negative [HbAA], sickle cell trait [HbAS], or sickle cell disease [HbSS]).

Auxiliary variables. Auxiliary variables that were used in the multiple imputation model included mother's height, birth rank, and gestational age. Mother's height was a dichotomous variable indicating less than $158 \mathrm{~cm}$ or $158 \mathrm{~cm}$ or more, as maternal height $<158 \mathrm{~cm}$ may increase the risk of intrauterine growth restriction. ${ }^{59}$ Birth rank was a dichotomous variable indicating the child's rank at birth (singleton or twin). Gestational age was an ordinal variable indicating weeks of gestational age at birth.

Statistical analysis. Multinomial generalized estimating equation models with an independent working correlation structure were used to estimate the association between

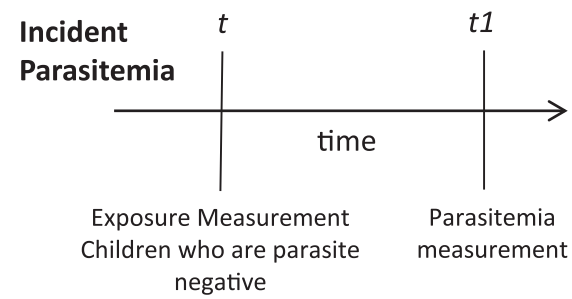

Figure 1. Time line of exposure and outcome measurements. For prevalent parasitemia, all children were included in the analysis, and the outcome was measured 1 month after the exposure. For incident parasitemia, only children with a negative blood film at time $t$ ( 1 month before the outcome measurement) were included in the analysis. 

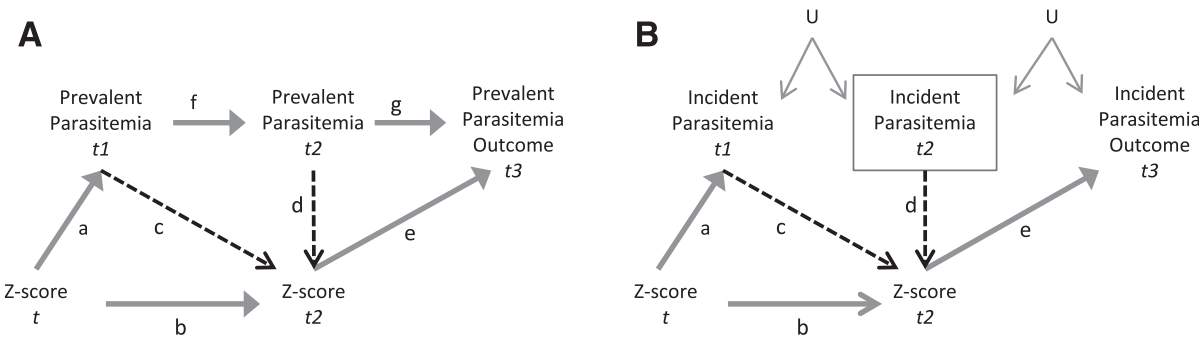

FIGURE 2. Directed acyclic graphs showing the relationship between Z-score and prevalent parasitemia (A), and Z-score and incident parasitemia (B). The yellow lines are paths we want to leave open because they are on the causal path from the exposure to the outcome. Red lines are associations that will be removed through inverse probability of exposure weights. $t$ is an indicator of time in months. $U$ is an unmeasured confounder that creates a potential link between two separate incident malaria parasite infections. In Figure A, prevalent parasitemia at one time point may be associated with prevalent parasitemia at a subsequent time point because it may either be the same, unresolved, untreated infection (because afebrile infections were untreated), or it may be a recrudescent, treated infection (because febrile infections were treated). Unmeasured confounders are not depicted in Figure A because there is already a direct link between subsequent prevalent malaria infections.

malnutrition and subsequent parasitemia while accounting for repeated observations on the same children over time. ${ }^{60}$ First, an unweighted model was used that adjusted for gender, season, mother's education, age (time), sector of residence, maternal peripheral parasitemia, sickle cell genotype, and non-malarial anemia and non-malarial illness in the month before the exposure. This model provided estimates for odds ratios (OR) comparing odds of parasitemia incidence or prevalence across different levels of stunting, under/ overweight, and wasting. Effect measure modification by non-malarial anemia and age was also explored. Results from interaction models were compared with the single effect models, and the interactions were assessed using Wald tests.

There were two types of missing data in the Asembo Bay Cohort Study completely missed visits, where no data were collected on a child for a particular month, and missing data within completed visits, where some data were collected on a child in a particular month, but not all. To address these two types of missing data, multiple imputation methods were combined with inverse probability weighting methods to provide effect estimates that were potentially less biased than a complete case analysis. ${ }^{61}$

To address missing data values within completed visits, multiple imputation by chained equations (MICE) was used for all variables included in the analysis ${ }^{62}$ (see Supplement B). Multiple imputation by chained equations is a flexible method of multiple imputation that allows for different imputation models for each variable with missing values. For each variable with missing data, 40 cycles of imputation were carried out before making one imputed dataset. In each cycle, each variable with missing values was regressed on all other variables, and missing values were replaced by simulated draws from the posterior predictive distribution of the missing variable. ${ }^{63} \mathrm{~A}$ total of 50 separate datasets were imputed. Each variable in the analysis was used to predict all other variables with the following exceptions: HAZ was used as a predictor for WAZ and vice versa, but neither was used as a predictor for WHZ because of collinearity concerns (see Tables 1 and 2 for information on which variables were imputed).

TABLE 1

Asembo Bay cohort study sample characteristics, Nyanza Province, Kenya, 1992-1996

\begin{tabular}{|c|c|c|c|}
\hline \multicolumn{4}{|c|}{ Children ages 0-30 months } \\
\hline \multirow[b]{2}{*}{ Variable } & \multicolumn{2}{|c|}{ Original data $(N=1,182)$} & \multirow{2}{*}{$\frac{50 \text { Datasets with imputed data }(N=59,100)}{N(\%) \text { or mean }(\mathrm{SE})}$} \\
\hline & $N(\%)$ or mean (SE) & Missing $(\%)^{\star}$ & \\
\hline Gender & - & 0 & - \\
\hline Male & $598(50.6)$ & - & $29,900(50.6)$ \\
\hline Female & $584(49.4)$ & - & $29,200(49.4)$ \\
\hline Age at enrollment (days) & $5.4(28.2)$ & 0 & $5.4(28.2)$ \\
\hline SÉS (value of goods, animals, and houses) & - & $4(0.33)$ & - \\
\hline Level 1 (lowest SES) & 588 (49.9) & - & $29,513(49.9)$ \\
\hline Level 2 (highest SES) & $590(50.1)$ & - & $29,587(50.1)$ \\
\hline Mother's education (years) & - & $4(0.33)$ & - \\
\hline$<7$ & $454(38.5)$ & - & $22,758(38.5)$ \\
\hline$\geq 7$ & $724(61.5)$ & - & $36,342(61.5)$ \\
\hline Village residence (sector) & - & $3(0.25)$ & - \\
\hline 1 & 380 (32.2) & - & $19,047(32.2)$ \\
\hline 2 & $315(26.7)$ & - & $15,787(26.7)$ \\
\hline 3 & $283(24.0)$ & - & $14,183(24.0)$ \\
\hline 4 & $201(17.1)$ & - & $10,083(17.1)$ \\
\hline Maternal peripheral parasitemia & - & $22(1.9)$ & - \\
\hline $\mathrm{Y}$ & $407(35.1)$ & & $20,738(35.1)$ \\
\hline $\mathrm{N}$ & 753 (65.0) & & $38,362(65.0)$ \\
\hline Sickle cell genotype & - & $173(14.6)$ & - \\
\hline Homozygous (SS) & $36(3.6)$ & - & $2,122(3.6)$ \\
\hline Heterozygous (AS) & $173(17.2)$ & - & $10,310(17.5)$ \\
\hline Negative (AA) & $800(79.3)$ & - & $46,668(79.0)$ \\
\hline
\end{tabular}

*The missing column indicates the number (\%) of children for whom missing baseline data were imputed. Gender and age were complete variables and were not imputed. 
TABLE 2

Asembo Bay cohort study sample characteristics, Nyanza Province, Kenya, 1992-1996

Children ages $0-30$ months $(n=1,182)$

\begin{tabular}{|c|c|c|c|}
\hline \multirow[b]{2}{*}{ Variable } & \multicolumn{2}{|c|}{ Observed data visits $=18,028$} & \multirow{2}{*}{$\frac{50 \text { Datasets with imputed data visits }=901,400}{N(\%) \text { or mean (SD) }}$} \\
\hline & $N(\%)$ or mean (SE) & Missing, $n(\%)$ & \\
\hline Height-for-age $Z$ score & $-1.57(0.01)$ & 4,934 (27.4) & $-1.61(0.01)$ \\
\hline Weight-for-age $Z$ score & $-0.45(0.01)$ & $4,353(24.2)$ & $-0.46(0.01)$ \\
\hline Weight-for-height $Z$ score & $0.63(0.01)$ & $5,073(28.1)$ & $0.63(0.01)$ \\
\hline Non-malarial illness* & $5,225(37.5)$ & $4,106(22.8)$ & $414,982(46.0)$ \\
\hline Non-malarial anemia* & $1,434(10.5)$ & $4,342(24.1)$ & $152,618(16.9)$ \\
\hline Febrile parasitemia prevalence & $3,123(21.3)$ & $3,375(18.7)$ & - \\
\hline Afebrile parasitemia prevalence & $6,365(43.4)$ & $3,375(18.7)$ & - \\
\hline Febrile parasitemia incidence & $1,400(9.6)$ & $3,375(18.7)$ & - \\
\hline Afebrile parasitemia incidence & $2,452(16.7)$ & $3,375(18.7)$ & - \\
\hline
\end{tabular}

In addition to the covariates and the outcome from the analysis model, other variables that predicted the incomplete variables and whether they were missing (auxiliary variables) were also included in the imputation models. ${ }^{63}$ Gestational age, birth rank, and mother's height were chosen as auxiliary variables based on their association with either the exposure or the outcome, or missingness of the exposure or the outcome. All three were included as auxiliary variables for all models, except for models imputing parasitemia variables and non-malarial anemia, in which only gestational age and birth rank were used. To take advantage of the longitudinal structure of the models, for each time-varying variable, the two previous measurements and two following measurements were included as auxiliary variables. ${ }^{64,65}$ All Z-score variables were imputed as continuous variables using predictive mean matching models, which provided imputed values that were consistent with observed values. ${ }^{66}$ Parasitemia variables and non-malarial anemia were imputed as categorical/binary variables in their final analytic form using logistic models. Logistic models for non-malarial anemia and parasitemia variables used the data augmentation method to account for perfect prediction when the maximum likelihood parameter estimates did not exist. In this method, the data were augmented with a small number of additional observations that were weighted to limit their impact on the imputation model. The addition of the augmenting variables averted perfect prediction and allowed the imputation model to converge. ${ }^{67}$ The discriminant function method (the default method for classification variables) was used to impute all other variables. ${ }^{68}$ Parasitemia outcome variables were included in the imputation models, but only observed outcomes were used in the final analysis models. ${ }^{69}$

The purpose of the study was to assess the association between malnutrition and subsequent malaria parasitemia. However, because malnutrition may also result from previous episodes of parasitemia, analytic techniques that allowed for the estimation of the association between malnutrition and subsequent parasitemia while preventing confounding by previous parasitemia episodes were necessary. Figure 2 depicts a DAG showing the relationship between Z-score and prevalent parasitemia (A), and Z-score and incident parasitemia (B). An arrow between two variables indicates that one variable directly affects the other variable (e.g., in Figure 2A, arrow $a$ indicates that $Z$-score at time $t$ affected prevalent parasitemia at time 11 ). The purpose of this study was to estimate the association between Z-score and subsequent parasitemia. Therefore, we needed to leave open all the paths from the exposure to the outcome (including those that pass through intermediate variables), indicated by yellow arrows in Figure 2.

In the prevalence models, previous and concurrent parasitemia status may have affected both current $Z$-score (the primary exposure) and future parasitemia status (the primary outcome), making previous parasitemia status a time-varying confounder affected by prior exposure (see Figure 2). In this model, previous parasitemia may be on a causal path from previous $Z$-score at time $t$ through parasitemia at time $t 1$ to parasitemia at time $t 2$ to the outcome at time $t 3$ (arrows $a$, $f$ and g). Inverse probability of exposure weights (IPEW) was used to remove the association between parasitemia at times $t 1$ and $t 2$ with $Z$-score at time $t 2$ (arrows $c$ and $d$ ) in the weighted analysis models. This removed potential bias due to time varying confounding by previous parasitemia status, while also keeping all causal paths open.

Likewise, the incident parasitemia models may also have been confounded by previous parasitemia status affected by prior exposure (Figure 1B). In the incident parasitemia models, we restricted the analysis to children with a negative blood film in the month before the outcome measurement (indicated by a box around parasitemia at time t2). In this model, parasitemia at subsequent time points was only associated through $U$, an unmeasured confounder linking the likelihood of a previous malaria parasite infection to future incidence, such as standing water outside the house. Malaria transmission is strongly associated with location, although the strength of this association decreases as transmission intensity increases. ${ }^{70}$ However, it has been shown that household location can be a predictor of variations in malaria incidence in children, even in high-transmission areas. ${ }^{71}$ It is important to consider that separate, incident parasitemia infections may have been associated through an unmeasured confounder such as $U$. By including only children with a negative blood film at time $t 2$ in the model, we open up a backdoor path from the exposure to the outcome through parasitemia at time $t 1$ and $U$. To avoid potential bias introduced by previous parasitemia status, we again used IPEW to remove the association between incident malaria at times $t 1$ and $t 2$ with $Z$-score at time $t 2$ (arrows $c$ and $d)$. 
Three different IPEWs were created-one for each type of anthropometric exposure (HAZ, WAZ, and WHZ). The use of IPEWs made the exposure in the weighted dataset independent of measured confounders, including previous and concurrent parasitemia status (see Figure $1 \mathrm{~A}$ and $\mathrm{B}$, times $t 1$ and $t 2$ ). Inverse probability of exposure weights were calculated as the inverse probability of exposure to Z-score deciles. ${ }^{72}$ Ordinal logistic regression was used to calculate the denominator of the weights as the probability of each child's observed Z-score decile in each month, conditional on all time-fixed covariates and time-varying covariates, including previous and concurrent parasitemia status. The weights were stabilized by the marginal probability of each child's observed $Z$-score decile, conditional on age. Weights were truncated at the first and 99th percentiles. ${ }^{73}$

To account for potential selection bias arising from missed visits, inverse probability of observation weights (IPOWs) were calculated with a logistic model for the inverse probability of completing a visit or having an observed outcome, conditional on predictors of completing a visit or having an observed outcome. ${ }^{74}$ Predictors included the following time-varying variables: observation history (a categorical indicator of months since last visit), exposure (HAZ, WAZ, and WHZ), age (modeled as a cubic variable, based on likelihood ratio tests), non-malarial illness, non-malarial anemia, and season. The following time-fixed variables were also used in the IPOW models: gender, residence, mother's parasitemia, mother's education, and sickle cell genotype. Time-varying variables were the last recorded value before the visit or outcome that was missed. Inverse probability of observation weights were stabilized by observation history, exposure, age, and baseline covariates mentioned earlier. Inverse probability of observation weights were then combined with inverse probability of censoring weights (IPCWs), which addressed potential selection bias from right censoring at last study encounter due to dropout. $^{75-77}$ Variables in the IPCW logistic model included exposures (HAZ, WAZ, and HWZ), age (modeled as a cubic variable, based on likelihood ratio tests), non-malarial illness, non-malarial anemia, season, residence, mother's education, mother's parasitemia, and sickle cell genotype. Inverse probability of censoring weights were stabilized by exposures, time-fixed covariates, and age. Final analysis results were similar whether deaths were included or excluded from the IPCWs. The final IPCWs used in the analysis models do not include weighting for death. Administrative censoring was not found to be differential with respect to the exposures or outcome, and therefore was also not included in the IPCWs. Inverse probability of exposure weights, IPOWs, and IPCWs were multiplied together into a final weight for each record and used to weight the final prevalence exposure outcome model for the multiply imputed data. ${ }^{61}$ The weight used for each analysis was dependent on the exposure in each model (HAZ, WAZ, or WHZ).

All analyses were conducted in SAS version 9.4 (SAS Institute, Cary, NC). The protocol was reviewed and approved by the CDC (\#1555) and the Ethical Review Board within the Kenya Medical Research Institute.

\section{RESULTS}

Study population. The original study included 1,408 infants and their siblings with follow-up data. Enrolled infants were followed up from birth through 4 years of age. The analysis sample was limited to the first child from each mother enrolled in the study, and follow-up was stopped at 30 months, when only $20 \%$ of the original sample remained. This resulted in a sample of 1,182 children followed up from age 0 to 30 months. Of these, 1,176 were enrolled within 5 months of birth, six were enrolled between 6 and 12 months, and one enrolled at 21 months. The mean age at the start of the study was 0.18 months (5.4 days) $(S D=0.94)$, and the mean age at the end of the study (average follow-up time) was 16.4 (SD =10.2) months. A total of 18,028 visits were recorded among the 1,182 children in the study, and $72 \%$ had at least one episode of febrile parasitemia, $79 \%$ had at least one episode of afebrile parasitemia, 64\% had at least one episode of each (febrile and afebrile parasitemia events), and $13 \%$ had neither. Sixty percent of study participants had an episode of non-malarial anemia, and $92 \%$ had at least one non-malarial infection. Prevalent parasitemia analyses were limited to records with an observed parasitemia outcome, which included 13,240 visits on 1,119 children. Incident parasitemia analyses were further limited to records with a negative blood film in the month before the outcome measurement, and included 4,020 observations on 995 children.

Missing data. Multiple imputation was used to impute missing values for the following baseline variables: residence sector, maternal peripheral parasitemia, maternal education, SES, and sickle cell genotype (see Table 1). Among completed visits, the following time-varying variables were also imputed: non-malarial illness, non-malarial anemia, HAZ, WAZ, and WHZ (see Table 2). Gender, age, and season were complete variables, and were not imputed. Time-fixed (Table 1) and time-varying (Table 2) study sample characteristics, including the percentage of missing variables that were imputed, and the distribution of imputed variables are presented in the following text.

Inverse probability of observation weights were calculated to account for 2,338(11\%) missed visits and 4,788 (24\%) visits with missing outcome data (IPOWs accounted for a total of 7,126 [35\%] missed visits). Of the 1,182 study subjects, 501 (42\%) terminated participation before the age of 30 months or at the end of the study, and 209 (18\%) died. Inverse probability of censoring weights were calculated to account for right censoring due to dropout among all 18,028 visits. Although death was associated both with febrile parasitemia (incidence rate ratio $=1.7,95 \%$ confidence limit $[C L]=1.6-1.7)$ and malnutrition $(\mathrm{HAZ} O \mathrm{OR}=2.08,95 \% \mathrm{CL}=1.12-3.87$; WAZ OR $=$ 6.02, 95\% CL = 3.08-11.79); $\mathrm{WHZ}$ OR $=7.1795 \% \mathrm{CL}=$ 2.43-21.23, IPCWs were not used to account for loss to follow-up due to death. The use of IPCWs to account for death would have approximated a study in which no children died. This would be an unreasonable assumption, given the population and time of the study. In addition, although death was associated with both the exposure and the outcome, the confounding path through death is blocked, because both malnutrition and malaria affect death, making death a variable we would not want to adjust for in traditional analyses. Last, results of the final analysis models did not differ significantly when deaths were included in the IPCWs. The combined final weights were well behaved, with means close to one and range not exceeding 0.2-3.4.

In general, there was not a strong association between any type of malnutrition and either prevalent or incident 


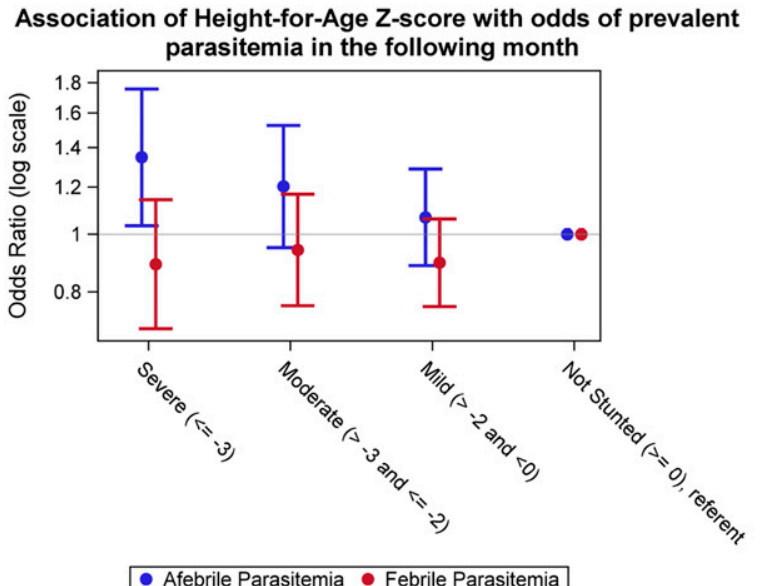

- Afebrile Parasitemia • Febrile Parasitemia

Association of Weight-for-Age Z-score with odds of prevalent parasitemia in the following month

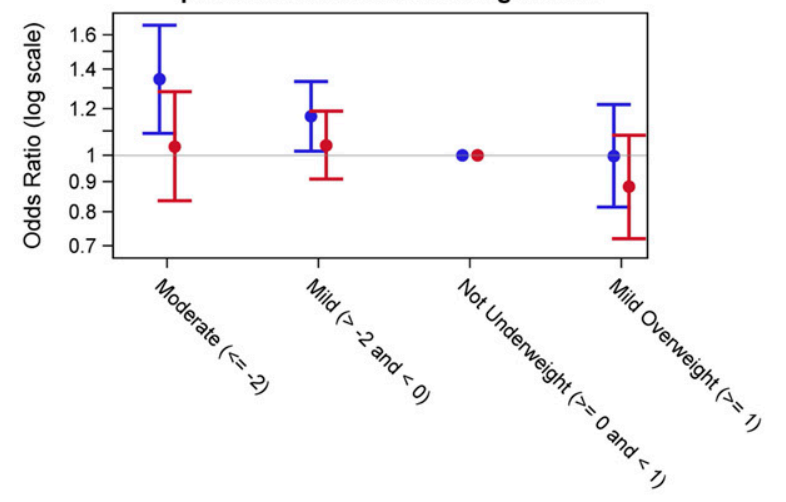

- Afebrile Parasitemia • Febrile Parasitemia

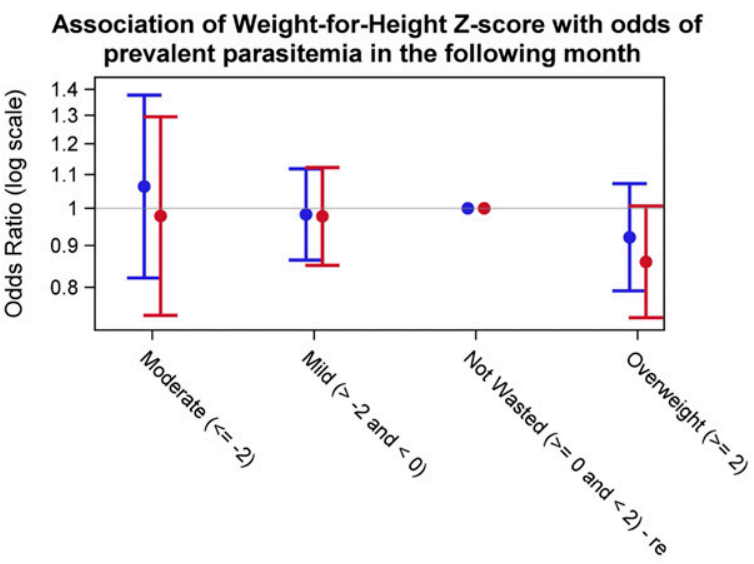

- Afebrile Parasitemia - Febrile Parasitemia
Association of Height-for-Age Z-score with odds of incident parasitemia in the following month

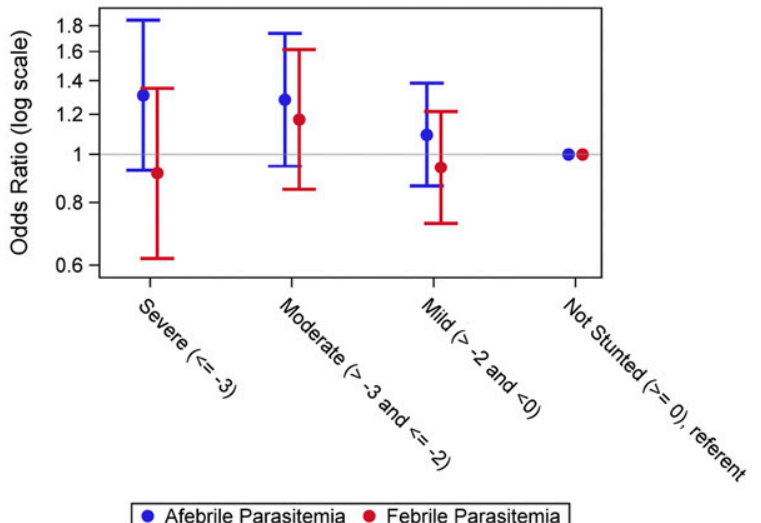

Association of Weight-for-Age Z-score with odds of incident parasitemia in the following month

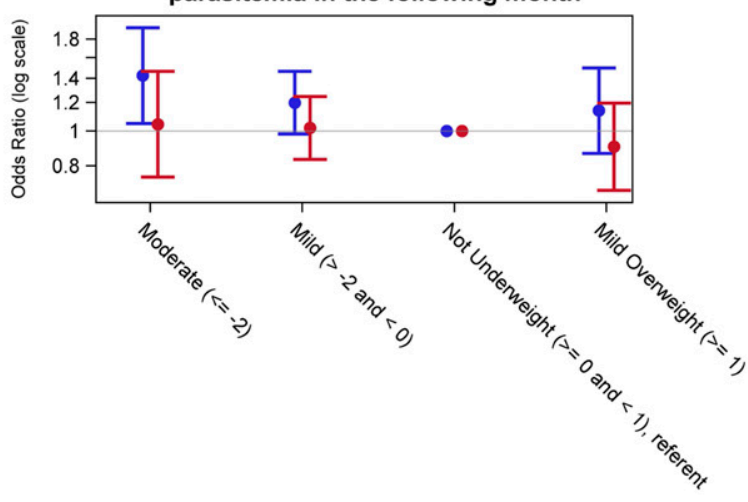

- Afebrile Parasitemia • Febrile Parasitemia

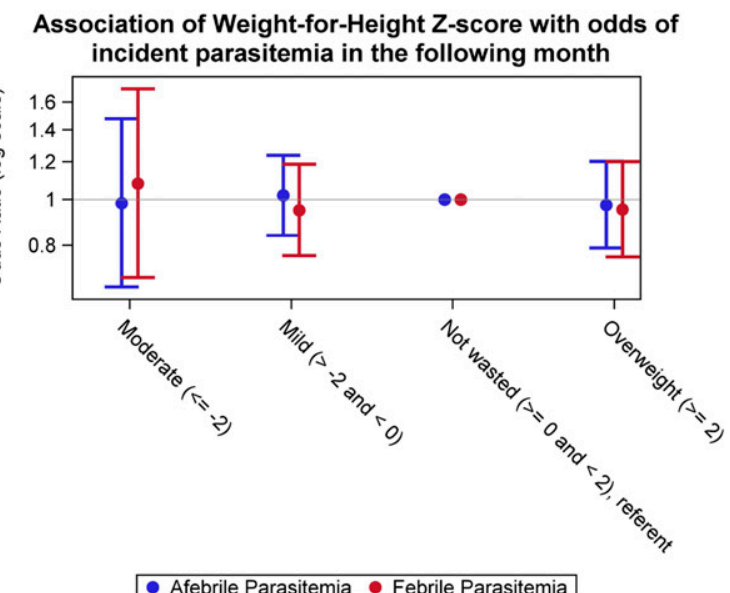

FIGURE 3. Associations among height-for-age, weight-for-age, and weight-for-height $Z$-score categories, and prevalent and incident malaria parasitemia outcomes in the following month. Odds ratios are from multiply imputed data with observed outcomes analyzed with weighted generalized estimating equation models. Prevalence models are shown in column $1, N=1,119$. Incidence models were limited to observations with a negative malaria parasite film in the month before the outcome. $N=995$. This figure appears in color at www.ajtmh.org.

parasitemia. Odds ratios for the association of stunting or underweight on prevalent and incident parasitemia were higher for afebrile parasitemia than for febrile parasitemia. This trend was more pronounced in the prevalence models. The association between stunting or underweight and prevalent afebrile parasitemia also showed a modest dose response, with prevalence ORs (PORs) increasing as Z-score decreased (see Figure 3). 
Reported results are from the weighted, imputed data analysis. The complete case analysis models were only slightly further from the null than the weighted, imputed data analysis (see Supplemental Table 1). These differences were not significant. Alternate time lags between the exposure and the outcome of 2 and 3 months were also explored. The results from these models were attenuated toward the null and have not been reported here.

Across both incident and prevalent malaria models, there was a modest association among stunting and underweight and afebrile parasitemia. The effects were slightly larger in the prevalence models. The POR for the association between severe or moderate stunting (HAZ) and prevalent afebrile parasitemia in the following month was as follows: severe = 1.35 (95\% CL: $1.03,1.76)$ and moderate $=1.20$ (95\% CL: 0.95 , 1.53). The POR for the association between moderate or mild WAZ and subsequent prevalent afebrile parasitemia was as follows: moderate $=1.35$ (95\% CL: $1.09,1.66)$ and mild $=1.16$ (95\% CL: 1.02, 1.33). The OR for the association between stunting and incident afebrile parasitemia was as follows: severe $=1.31(95 \%$ CL: $0.93,1.85)$ and moderate $=1.28(95 \%$ CL: $0.95,1.74)$. For underweight, the OR for incident afebrile parasitemia was as follows: moderate $=1.43$ (95\% CL: 1.05 , 1.94) and mild = 1.20 (95\% CL: 0.98, 1.46).

There was no significant association between any category of wasting and prevalent or incident malaria in the following month. There was also no association seen between any type of malnutrition and either type of febrile parasitemia in the following month (prevalent or incident).

\section{DISCUSSION}

Using data from the Asembo Bay cohort study in western Kenya, we found that stunting and underweight were associated with slightly increased odds of subsequent prevalent and incident afebrile parasitemia in children younger than 30 months. However, there was no evidence of an association between any measure of malnutrition and either type of febrile parasitemia in the following month, nor was there an association between wasting and any type of incident or prevalent malaria.

The null association between malnutrition and febrile parasitemia is consistent with most of the previous longitudinal studies, which also found that malnutrition did not increase the risk of malaria with fever. ${ }^{10,13,15-18}$ Two previous studies did find an increased incidence of febrile malaria among stunted (moderate-severe) children compared with non-stunted children. ${ }^{11,12}$ However, neither adjusted for non-malarial infections, which may modulate the response to malaria. ${ }^{78}$ In addition, both could be subject to reverse causality bias, given that exposures were either measured at the same time as the outcome $^{12}$ or only at baseline (and therefore might have changed during the follow-up period). ${ }^{11}$

There are few previous studies on the association between malnutrition and asymptomatic parasitemia. One study showed an association between stunting and concurrent asymptomatic parasitemia, ${ }^{23}$ and two studies found no association. ${ }^{14,24}$ An increased OR for afebrile parasitemia, although not for febrile parasitemia, was an unexpected finding in this study. A later cross-sectional study conducted in the same region among children who received ITNs also found a higher OR for the association between moderate stunting and any parasitemia (1.98) than for stunting and clinical malaria (1.77). ${ }^{79}$

It is unclear why underweight and stunted children in this population would have increased odds of afebrile infection, but not febrile infection. The paucity of previous research with afebrile or asymptomatic infections as an outcome makes it difficult to determine if this was a regularly occurring phenomenon within areas of intense malaria transmission, or something particular to this population. The relationship between malnutrition and malaria is complex, and findings from other studies are potentially dependent on the epidemiology of malaria and the determinants of malnutrition specific to each study population. Nevertheless, we can develop hypotheses based on our current knowledge of the immune system among children with malnutrition. One possible explanation may be immune dysfunction among chronically malnourished children. There is limited research on the effects of malnutrition on the immune system, including the development of fever. A recent review highlighted that the Type $1 \mathrm{~T}$ helper cells inflammatory response and the number of activated dendritic cells tend to be reduced in malnourished children compared with well-nourished children, whereas the type $2 \mathrm{~T}$ helper cells response is increased in moderately malnourished children. ${ }^{80} \mathrm{~A}$ lowered response by Type $1 \mathrm{~T}$ helper cells cytokines and dendritic cells among chronically malnourished children could potentially lead to a reduced febrile response to malaria parasites in this group, resulting in higher odds of afebrile parasitemia.

Alternatively, immune exhaustion may play a role in increasing odds of afebrile parasitemia among stunted and underweight children. Fontana discussed the role that exhausted T cells may play in asymptomatic parasitemia. She suggests that these exhausted T cells may be mediators of the relationship between the host and the pathogen during chronic infection, whereby exhausted T cells fail to clear the infection yet can limit the parasite load to avoid "overwhelming pathology to the host." ${ }^{81}$ Chronic malnutrition such as stunting or underweight may have a larger effect on afebrile parasitemia rather than febrile parasitemia if malnourished children are more likely to experience immune exhaustion placing them at higher risk of afebrile parasitemia, whereby the parasites are regulated by exhausted T cells.

The wasting exposure models did not show an association with subsequent incident or prevalent parasitemia. This is consistent with most of the longitudinal studies that also found no association between wasting and malaria outcomes. ${ }^{11,15,16,18,82}$ Wasting and stunting represent different forms of malnutrition. Wasting represents acute malnutrition, often occurring over a short period of time, whereas stunting represents chronic malnutrition, developing over a longer period of time. It should also be noted that stunting could be the result of a previous episode of growth faltering that the child has failed to recover from. These different forms of malnutrition may have different effects on the immune response to malaria. When comparing malnourished children with non-stunted or non-wasted controls, Fillol et al. ${ }^{83}$ found the adaptive immune response to malaria was significantly lower in stunted children but did not see this effect in wasted children. No studies have specifically investigated the effect of malnutrition on the innate immune response to malaria. Our study only investigated the association of moderate wasting $(\mathrm{WHZ} \leq-2)$ with subsequent malaria outcomes, due to a limited number of children with severe wasting $(\mathrm{WHZ} \leq-3)$. It is 
possible that severe wasting could show an association with subsequent malaria, due to the effects severe wasting can have on immune function and its association with increased mortality. ${ }^{84}$

\section{STRENGTHS AND LIMITATIONS}

This study had several strengths and limitations. Strengths included a large sample of children followed from birth with data on both febrile parasitemia and afebrile parasitemia, biweekly blood collections, and monthly anthropometric measurements, and the availability of a wide-ranging set of potential confounders to use in multivariable models. Although the dataset is older, the higher incidence of malaria that occurred in this study population than current populations likely provided the study provide greater power to detect these associations than a similar study conducted in a present day population benefiting from broad scale-up of both preventive and curative malaria interventions.

The age of the dataset makes the study less generalizable to most current populations across Africa. The current epidemiology of malaria in western Kenya may be different from that experienced by this cohort. Since 2000 , incidence of malaria in Kenya has decreased from $237.7 / 1,000$ people at risk to 166/ 1,000 , and the prevalence of children sleeping under ITNs has increased from $3 \%$ to $56.1 \% .{ }^{85}$ Associations detected between malnutrition and malaria in this 1992-1996 dataset may not necessarily represent associations that currently exist in western Kenya if the relationship between malaria and nutrition is influenced by the specific epidemiology of malaria. In addition, differences in historic and current malaria treatment may also make the findings less generalizable. The close follow-up of participants, prompt diagnosis, treatment with SP, and protocol for treatment failures (halofantrine) should have ensured that all children were treated adequately for malaria, while minimizing and managing any treatment failures during this era of decreased chloroquine efficacy. ${ }^{86}$

There have also been improvements in health and nutrition status of children younger than 5 years over the past 25 years. From 1998 to 2014, the prevalence of stunting in Kenya decreased from $37 \%$ to $26 \%$. Likewise, optimal child feeding practices such as exclusive breastfeeding have improved. ${ }^{87,88}$ As children's health and nutrition have improved, and malaria incidence has declined, the relationship between malnutrition and malaria may have changed. Proximal determinants of malnutrition may have changed with improvements in nutrition practices and sanitation, which may, in turn, have an effect on children's immune systems, rendering them more or less susceptible to malaria. Likewise, changes in the epidemiology of malaria, such as increasing age of acquired immunity, and decreasing attack rates may change how malnourished children respond to malaria.

This study used low hemoglobin as a proxy for irondeficiency anemia. Low hemoglobin can be caused by iron deficiency, but also by various infections and other nutritional deficiencies, and is not an ideal proxy for iron-deficiency anemia. We increased the specificity of low hemoglobin as a marker for iron deficiency by excluding anemia within 1 month of malaria parasitemia, and controlling for sickle cell disease. It is possible that some non-malarial anemia in this study could still have been associated with previous repeated bouts of parasitemia. $^{89}$ Most previous studies of the relationship between malnutrition and subsequent malaria did not control for iron-deficiency anemia. ${ }^{11-15,17}$ The inclusion of the proxy measure as a confounder, although not ideal, is an improvement over previous research.

The high proportion of missing data in this study is a limitation. Measures were taken to minimize bias due to missing data and dropout, which included multiple imputation and the use of inverse probability weights to account for missed visits and dropout. However, if missingness was due to unobserved variables that were also associated with the exposure or the outcome, selection bias may still be present.

The evaluation of malaria was performed by thick and thin blood smears, which may have missed submicroscopic infections. This diagnostic limitation may have biased results toward null if the outcome misclassification was nondifferential with respect to malnutrition. Alternatively, if malnourished children were more likely to have microscopically identifiable parasites, then this would have biased the results away from null.

HIV may be a confounder of the relationship between both growth faltering and malaria, and data on HIV status were not available for this study. The prevalence of HIV among pregnant women at the time of the study was estimated at $30 \% .{ }^{90} \mathrm{HIV}$ may increase the prevalence of malaria in children ${ }^{91}$ or increase parasite density. ${ }^{92}$ If children with HIV were more likely to have afebrile parasitemia and be underweight or stunted, the ORs may have been biased away from the null. However, if HIV was associated with a measured confounder (such as non-malarial infection, SES, or mother's education) conditional on the exposure, confounding bias due to HIV may have been partially controlled by these other variables. ${ }^{93}$ The relationship between stunting and malaria has previously been shown to be consistent across HIV-infected and uninfected children. ${ }^{12}$ Given the demonstrated independence of the malnutrition-malaria relationship from HIV status and the potential for residual control by included covariates, the results of this study are unlikely to be significantly biased by unmeasured confounding from HIV. However, interactions between various coinfections that were prevalent in the area at this time, such as tuberculosis and HIV, and their potential to influence the malaria-malnutrition relationship cannot be ruled out. ${ }^{94-96}$

The potential cyclical nature of the relationship between malnutrition and malaria makes epidemiologic research on this topic challenging. Malnutrition may increase the risk of malaria, whereas malaria may also increase the risk of malnutrition. The use of IPEWs to adjust for time-varying confounding affected by prior exposure allowed for an estimation of the association while preventing potential reverse causality bias due to prior malaria infection. The use of these methods makes this study a novel addition to the literature on this complex relationship. The similarities between the complete case analysis and the imputed, weighted model indicate that missing data and time-varying confounding were not a large source of bias in this population.

\section{CONCLUSION}

Stunting and underweight do not show an association with febrile parasitemia, but they do show a limited association with afebrile parasitemia in the month following the exposure. This association was seen even in mildly underweight children. 
Current malaria control efforts may consider prioritizing test and treat strategies for specific populations of children at greater risk of stunting and underweight. Malaria testing for stunted and underweight children in malaria endemic areas should be considered, even if children do not present with fever.

Received January 2, 2020. Accepted for publication August 22, 2020.

Published online November 16, 2020.

Note: Supplemental materials and tables appear at www.ajtmh.org.

Disclosure: The findings and conclusions in this report are those of the author(s) and do not necessarily represent the official position of the Centers for Disease Control and Prevention.

Authors' addresses: Catherine V. Donovan, Brian W. Pence, and Steven Meshnick, Department of Epidemiology, University of North Carolina at Chapel Hill, Chapel Hill, NC, E-mails: cvdonovan@ alumni.unc.edu, and bpence@unc.edu. Peter McElroy, Division of Tuberculosis Elimination, Centers for Disease Control and Prevention, Atlanta, GA, E-mail: pgm9@cdc.gov. Linda Adair, Nutrition, University of North Carolina Chapel Hill, Chapel Hill, NC, E-mail: linda_adair@ unc.edu. Aggrey James Oloo, Department of Medicine, Masinde Muliro University of Science and Technology, Kakamega, Kenya, E-mail: olooaj@yahoo.co.uk. Altaf Lal, Global Health and Innovation, Sun Pharmaceutical Industries Ltd, Health and Innovation, Mumbai, India, E-mail: altaf.lal@gmail.com. Peter Bloland, Division of Parasitic Diseases, Centers for Disease Control and Prevention, Atlanta, GA, E-mail: pbb1@cdc.gov. Bernard Nahlen, Eck Institute for Global Health, University of Notre Dame, Notre Dame, IN, E-mail: bnahlen@ nd.edu. Jonathan J. Juliano, Division of Infectious Diseases, Department of Medicine, University of North Carolina at Chapel Hill, Chapel Hill, NC, E-mail: jonathan_juliano@med.unc.edu.

\section{REFERENCES}

1. WHO, 2019. World Malaria Report 2019. Geneva, Switzerland: World Health Organization. Available at: https://www.who.int/ publications/i/item/world-malaria-report-2019. Accessed August $9,2020$.

2. UNICEF, 2019. Children in Africa: Key Statistics on Child Survival and Population. Available at: https://data.unicef.org/resources/ children-in-africa-child-survival-brochure/. Accessed August 9, 2020.

3. United Nations Children's Fund, World Health Organization, World Bank Group, 2020. Levels and Trends in Child Malnutrition: Key Findings of the 2020 Edition. Geneva, Switzerland: UNICEF. Available at: https://www.unicef.org/reports/joint-child-malnutritionestimates-levels-and-trends-child-malnutrition-2020. Accessed August 9, 2020.

4. Development Initiatives, 2017. Global Nutrition Report 2017: Kenya Nutrition Country Profile. Bristol, United Kingdom: Development Initiatives.

5. Schroeder DG, 2008. Malnutrition. Semba RD, Bloem MW, eds. Nutrition and Health in Developing Countries, 2nd edition. Totowa, NJ: Humana Press.

6. Hess FI, Nukuro E, Judson L, Rodgers J, Nothdurft HD, Rieckmann KH, 1997. Anti-malarial drug resistance, malnutrition and socio-economic status. Trop Med Int Heal 2: 721-728.

7. Bresnahan KA, Tanumihardjo SA, 2014. Undernutrition, the acute phase response to infection, and its effects on micronutrient status indicators. Adv Nutr 5: 702-711.

8. Stevenson MM, Riley EM, 2004. Innate immunity to malaria. Nature 4: 169-180.

9. Das D, Grais RF, Okiro EA, Stepniewska K, Mansoor R, van der Kam S, Terlouw DJ, Tarning J, Barnes KI, Guerin PJ, 2018. Complex interactions between malaria and malnutrition: a systematic literature review. BMC Med 16: 186.

10. Tonglet $R$, Mahangaiko Lembo E, Zihindula PM, Wodon A, Dramaix M, Hennart P, 1999. How useful are anthropometric, clinical and dietary measurements of nutritional status as predictors of morbidity of young children in central Africa? Trop Med Int Heal 4: 120-130.
11. Deen J, Walraven G, Seidlein L, 2002. Increased risk for malaria in chronically malnourished children under 5 years of age in rural Gambia. J Trop Pediatr 48: 78-83.

12. Arinaitwe $\mathrm{E}$ et al., 2012. The association between malnutrition and the incidence of malaria among young HIV-infected and -uninfected Ugandan children: a prospective study. Malar J 11: 90.

13. Fillol F, Cournil A, Boulanger D, Cissé B, Sokhna C, Targett G, Trape JF, Simondon F, Greenwood B, Greenwood B, Simondon KB, 2009. Influence of wasting and stunting at the onset of the rainy season on subsequent malaria morbidity among rural preschool children in Senegal. Am J Trop Med Hyg 80: 202-208.

14. Genton B, Al-Yaman F, Ginny M, Taraika J, Alpers MP, 1998. Relation of anthropometry to malaria morbidity and immunity in Papua New Guinean children. Am J Clin Nutr 68: 734-741.

15. Snow RW, Byass $P$, Shenton FC, Greenwood BM, 1991. The relationship between anthropometric measurements and measurements of iron status and susceptibility to malaria in Gambian children. Trans $R$ Soc Trop Med Hyg 85: 584-589.

16. Williams TN, Maitland K, Phelps L, Bennett S, Peto TE, Viji J, Timothy R, Clegg JB, Weatherall DJ, Bowden DK, 1997. Plasmodium vivax: a cause of malnutrition in young children. $Q J$ Med 90: 751-757.

17. Danquah I, Dietz E, Zanger $P$, Reither $K$, Ziniel $P$, Bienzle U, Mockenhaupt FP, 2009. Reduced efficacy of intermittent preventive treatment of malaria in malnourished children. Antimicrob Agents Chemother 53: 1753-1759.

18. Oldenburg CE, Guerin PJ, Berthé F, Grais RF, Isanaka S, 2018. Malaria and nutritional status among children with severe acute malnutrition in Niger: a prospective cohort study. Clin Infect Dis 67: 1027-1034.

19. Ferreira A, Alexandre MA, Salinas JL, de Siqueira AM, Benzecry SG, de Lacerda MVG, Monteiro WM, 2015. Association between anthropometry-based nutritional status and malaria: a systematic review of observational studies. Malar J 14: 1-23.

20. Lindblade KA, Steinhardt L, Samuels A, Kachur SP, Slutsker L, 2013. The silent threat: asymptomatic parasitemia and malaria transmission. Expert Rev Anti Infect Ther 11: 623-639.

21. de Mast Q, Brouwers J, Syafruddin D, Bousema T, Baidjoe AY, de Groot PG, van der Ven AJ, Fijnheer R, 2015, Is asymptomatic malaria really asymptomatic? Hematological, vascular and inflammatory effects of asymptomatic malaria parasitemia. $J$ Infect 71: 587-596.

22. Chen I, Clarke SE, Gosling R, Hamainza B, Killeen G, Magill A, O'Meara W, Price RN, Riley EM, 2016. "Asymptomatic" malaria: a chronic and debilitating infection that should be treated. PLoS Med 13: e1001942.

23. Akiyama T et al., 2016. Asymptomatic malaria, growth status, and anaemia among children in Lao People's Democratic Republic: a cross-sectional study. Malar J 15: 499.

24. Crookston BT et al., 2010. Exploring the relationship between chronic undernutrition and asymptomatic malaria in Ghanaian children. Malar J 9: 39.

25. WHO, 2018. World Malaria Report 2018. Geneva, Switzerland: World Health Organization.

26. United Nations Children's Fund, World Health Organization, World Bank Group, 2018. Levels and Trends in Child Malnutrition: Key Findings of the 2018 Edition of the Joint Child Malnutrition Estimates. Available at: https://data.unicef.org/wp-content/ uploads/2018/05/JME-2018-brochure-web.pdf. Accessed January 6,2019 .

27. World Health Organization, Global Malaria Programme, 2018. RBM partnership to end malaria. High Burden to High Impact: A Targeted Malaria Response. Geneva, Switzerland: WHO. Available at: https://apps.who.int/iris/bitstream/handle/10665/ 275868/WHO-CDS-GMP-2018.25-eng.pdf?ua=1. Accessed January 29, 2019.

28. World Health Organization, 2014. Comprehensive Implementation Plan on Maternal, Infant and Young Child Nutirition. Geneva, Switzerland: WHO. Available at: https://apps.who.int/iris/bitstream/ handle/10665/113048/WHO_NMH_NHD_14.1_eng.pdf?ua=1. Accessed January 29, 2019.

29. Beier JC et al., 1994. Plasmodium falciparum incidence relative to entomologic inoculation rates at a site proposed for testing 
malaria vaccines in western Kenya. Am J Trop Med Hyg 50: 529-536.

30. Ministry of Health, Program NMC, 2016. The Epidemiology and Control Profile of Malaria in Kenya: Reviewing the Evidence to Guide the Future Vector Control. Nairobi, Kenya: National Malaria Control Programme. Available at: http://www.nmcp.or.ke. Accessed January 24, 2019.

31. Bloland PB et al., 1999. Longitudinal cohort study of the epidemiology of malaria infections in an area of intense malaria transmission I. Description of study site, general methodology, and study population. Am J Trop Med Hyg 60: 635-640.

32. van Schenck H, Falkensson M, Lundberg B, 1986. Evaluation of "HemoCue," a new device for determining hemoglobin. Clin Chem 60: 526-529.

33. Wu DY, Ugozzoli L, Pal BK, Wallace RB, 1989. Allele-specific enzymatic amplification of beta-globin genomic DNA for diagnosis of sickle cell anemia. Proc Natl Acad Sci U S A 86: 2757-2760.

34. WHO Multicentre Growth Reference Study Group, 2006. WHO Child Growth Standards: Length/Height-for-Age, Weight-forAge, Weight-for-Length, Weight-for-Height and Body Mass Index-for-Age: Methods and Development. Geneva, Switzerland: WHO. Available at: http://www.who.int/childgrowth/ standards/Technical_report.pdf?ua $=1$. Accessed April 27, 2015.

35. Thurber KA, Banks E, Banwell C, 2014. Approaches to maximising the accuracy of anthropometric data on children: review and empirical evaluation using the Australian longitudinal study of indigenous children. Public Heal Res Pr 25: 1-8.

36. Greenland S, Pearl J, Robins JM, 1999. Causal diagrams for epidemiologic research. Epidemiology 10: 37-48.

37. Nyakeriga AMM, Troye-Blomberg M, Dorfman JRR, Alexander ND, Bäck R, Kortok M, Chemtai AK, Marsh K, Williams TN, 2004. Iron deficiency and malaria among children living on the coast of Kenya. J Infect Dis 190: 439-447.

38. Clark MA, Goheen MM, Cerami C, 2014. Influence of host iron status on Plasmodium falciparum infection. Front Pharmacol 5: 84.

39. Pullan RL, Gitonga C, Mwandawiro C, Snow RW, Brooker SJ, 2013. Estimating the relative contribution of parasitic infections and nutrition for anaemia among school-aged children in Kenya: a subnational geostatistical analysis. BMJ Open 3: $1-10$.

40. Bloland PB, Boriga DA, Ruebush TK, McCormick JB, Roberts JM, Oloo AJ, Hawley W, Lal A, Nahlen B, Campbell CC, 1999. Longitudinal cohort study of the epidemiology of malaria infections in an area of intense malaria transmission II. Descriptive epidemiology of malaria infection and disease among children. Am J Trop Med Hyg 60: 641-648.

41. Sewe M, Rocklöv J, Williamson J, Hamel M, Nyaguara A, Odhiambo F, Laserson K, 2015. The association of weather variability and under five malaria mortality in KEMRI/CDC HDSS in western Kenya 2003 to 2008: a time series analysis. Int $J$ Environ Res Public Health 12: 1983-1997.

42. Prentice AM, Cole TJ, 1994. Seasonal changes in growth and energy status in the Third World. Proc Nutr Soc 53: 509-519.

43. Nacher M, Singhasivanon P, Treeprasertsuk S, Vannaphan S, Traore B, Looareesuwan S, Gay F, 2002. Intestinal helminths and malnutrition are independently associated with protection from cerebral malaria in Thailand. Ann Trop Med Parasitol 96: 5-13.

44. Dewey KG, Mayers DR, 2011. Early child growth: how do nutrition and infection interact? Matern Child Nutr 7 (Supp/3): 129-142.

45. Ibadin O, Airauhi L, Omoigberale A, Abiodun P, 2000. Association of malarial parasitaemia with dehydrating diarrhoea in Nigerian children. J Health Popul Nutr 18: 115-118.

46. Pan W, 2001. Akaike's information criterion in generalized estimating equations. Biometrics 57: 120-125.

47. Kepha S et al., 2016. Plasmodium falciparum parasitaemia and clinical malaria among school children living in a high transmission setting in western Kenya. Malar J 15: 157.

48. Ma C, Claude KM, Kibendelwa ZT, Brooks H, Zheng X, Hawkes M, 2017. Is maternal education a social vaccine for childhood malaria infection? A cross-sectional study from war-torn
Democratic Republic of Congo. Pathog Glob Health 111: 98-106.

49. Wanzira H, Katamba H, Okullo AE, Agaba B, Kasule M, Rubahika D, 2017. Factors associated with malaria parasitaemia among children under 5 years in Uganda: a secondary data analysis of the 2014 malaria indicator survey dataset. Malar J 16: 1-9.

50. Hoffman D, Cacciola T, Barrios P, Simon J, 2017. Temporal changes and determinants of childhood nutritional status in Kenya and Zambia. J Health Popul Nutr 36: 27.

51. Sultana M, Sheikh N, Mahumud RA, Jahir T, Islam Z, Sarker AR, 2017. Prevalence and associated determinants of malaria parasites among Kenyan children. Trop Med Health 45: 1-9.

52. Amek N, Bayoh N, Hamel M, Lindblade KA, Gimnig JE, Odhiambo F, Laserson KF, Slutsker L, Smith T, Vounatsou P, 2012. Spatial and temporal dynamics of malaria transmission in rural Western Kenya. Parasit Vectors 5: 86.

53. Homan T et al., 2016. Spatially variable risk factors for malaria in a geographically heterogeneous landscape, western Kenya: an explorative study. Malar J 15: 1.

54. Pawloski LR, Curtin KM, Gewa C, Attaway D, 2012. Maternalchild overweight/obesity and undernutrition in Kenya: a geographic analysis. Public Health Nutr 15: 2140-2147.

55. Bardají A, Sigauque B, Sanz S, Maixenchs M, Ordi J, Aponte JJ, Mabunda S, Alonso PL, Menéndez C, 2011. Impact of malaria at the end of pregnancy on infant mortality and morbidity. $J$ Infect Dis 203: 691-699.

56. Uneke C, 2007. Impact of placental Plasmodium falciparum malaria on pregnancy and perinatal outcome in sub-Saharan Africa: I: introduction to placental malaria. Yale $\mathrm{J} \mathrm{Biol} \mathrm{Med} \mathrm{80:}$ 39-50.

57. Allison AC, 1954. Protection afforded by sickle-cell trait against subtertian malarial infection. Br Med J 1: 290-294.

58. Platt OS, Rosenstock W, Espeland M, 1984. Influence of sickle hemoglobinopathies on growth and development. $N$ Engl J Med 311: 7-12.

59. Kramer MS, 1987. Determinants of low birth weight: methodological assessment and meta-analysis. Bull World Health Organ 65: 663-737.

60. Miller ME, Davis CS, Landis JR, 1993. The analysis of longitudinal polytomous data: generalized estimating equations and connections with weighted least squares. Biometrics 49: 1033-1044.

61. Seaman SR, White IR, Copas AJ, Li L, 2012. Combining multiple imputation and inverse-probability weighting. Biometrics 68: 129-137.

62. Van Buuren S, 2007. Multiple imputation of discrete and continuous data by fully conditional specification. Stat Methods Med Res 16: 219-242.

63. White IR, Royston P, Wood AM, 2011. Multiple imputation using chained equations: issues and guidance for practice. Stat Med 30: 377-399.

64. Biering $\mathrm{K}$, Hjollund $\mathrm{NH}$, Frydenberg M, 2015. Using multiple imputation to deal with missing data and attrition in longitudunal studies with repeated measures of patient reported outcomes. $J$ Clin Epidemiol 7: 91-106.

65. Fielding S, Fayers P, Ramsay C, 2010. Predicting missing quality of life data that were later recovered: an empirical comparison of approaches. Clin Trials 7: 333-342.

66. Schenker N, Taylor JMG, 1996. Partially parametric techniques for multiple imputation. Comput Stat Data Anal 22: 425-446.

67. White IR, Daniel R, Royston P, 2010. Avoiding bias due to perfect prediction in multiple imputation of incomplete categorical variables. Comput Stat Data Anal 54: 2267-2275.

68. Brand JPL, 1999. Development, Implementation and Evaluation of Multiple Imputation Strategies for the Statistical Analysis of Incomplete Data Sets. Rotterdam, The Netherlands: Print Partners Ispkamp, Enschede.

69. Von Hippel PT, 2007. Regression with missing Ys: an improved strategy for analyzing multiply imputed data. Sociol Methodol 37: 83-117.

70. Carter R, Mendis KN, Roberts D, 2000. Spatial targeting of interventions against malaria. Bull World Health Organ 78: 1401-1411.

71. Kreuels B, Kobbe R, Adjei S, Kreuzberg C, von Reden C, Bäter K, Klug S, Busch W, Adjei O, May J, 2008. Spatial variation of 
malaria incidence in young children from a geographically homogeneous area with high endemicity. J Infect Dis 197: 85-93.

72. Naimi Al, Moodie EEM, Auger N, Kaufman JS, 2014. Constructing inverse probability weights for continuous exposures. Epidemiology 25: 292-299.

73. Cole SR, Hernán MA, 2008. Constructing inverse probability weights for marginal structural models. Am J Epidemiol 168: 656-664.

74. Hernan MA, McAdams M, McGrath N, Lanoy E, Costagliola D, 2009. Observation plans in longitudunal studies with timevarying treatments. Stat Methods Med Res 18: 1-29.

75. Howe CJ, Cole SR, Lau B, Napravnik S, Eron JJ, 2016. Selection bias due to loss to follow up in cohort studies. Epidemiology 27: $91-97$.

76. Seaman SR, White IR, 2011. Review of inverse probability weighting for dealing with missing data. Stat Methods Med Res 22: 278-295.

77. Lesko CR, Edwards JK, Cole SR, Moore RD, Lau B, 2018. When to censor? Am J Epidemiol 187: 623-632.

78. Gonçalves RM, Lima NF, Ferreira MU, 2014. Parasite virulence, co-infections and cytokine balance in malaria. Pathog Glob Health 108: 173-178.

79. Friedman JF, Kwena AM, Mirel LB, Kariuki SK, Terlouw DJ, Phillips-Howard PA, Hawley WA, Nahlen BL, Shi YP, ter Kuile FO, 2005. Malaria and nutritional status among pre-school children: results from cross-sectional surveys in western Kenya. Am J Trop Med Hyg 73: 698-704.

80. Rytter MJH, Kolte L, Briend A, Friis H, Christensen VB, 2014. The immune system in children with malnutrition-a systematic review. PLoS One 9: e105017.

81. Fontana MF et al., 2016. A novel model of asymptomatic plasmodium parasitemia that recapitulates elements of the human immune response to chronic infection. PLoS One 11: e0162132.

82. Custodio E, Descalzo M, Villamor E, Molina L, Sánchez I, Lwanga M, Bernis C, Benito A, Roche J, 2009. Nutritional and socioeconomic factors associated with Plasmodium falciparum infection in children from Equatorial Guinea: results from a nationally representative survey. Malar $\mathrm{J}$ 8: 225.

83. Fillol F, Sarr JB, Boulanger D, Cisse B, Sokhna C, Riveau G, Simondon KB, Remoué F, 2009. Impact of child malnutrition on the specific anti-Plasmodium falciparum antibody response. Malar J 8: 116.

84. Bourke CD, Berkley JA, Prendergast AJ, 2016. Immune dysfunction as a cause and consequence of malnutrition. Trends Immunol 37: 386-398.
85. The World Bank, -. World Development Indicators. Available at: https://datacatalog.worldbank.org/dataset/world-developmentindicators. Accessed February 7, 2019.

86. Bloland PB, Ettling M, 1999. Making malaria treatment policy in the face of drug resistance. Ann Trop Med Parasitol 93: 5-23.

87. International Food Policy Research Institute, 2014. Global Nutrition Report 2014: Kenya Nutrition Country Profile. Washington, DC: International Food Policy Research Institute.

88. UNICEF, WHO, World Bank Group, 2017. Joint Child Malnutrition Estimates. Joint Child Malnutrition Estimates. Available at: http://www.who.int/nutgrowthdb/estimates2016/en/. Accessed February 7, 2019.

89. White NJ, 2018. Anaemia and malaria. Malar J 17: 371

90. Office of the President, Kenya, National AIDS Control Council, Ministry of Health, Kenya National AIDS/STD/TB/Leprosy Control Programme, MEASURE Evaluation, Population Services International, 2004. AIDS in Africa During the Nineties: Kenya. A review and analysis of survey and research results. Carolina Population Center, University of North Carolina, Chapel Hill: Chapel Hill, NC.

91. Flateau C, Le Loup G, Pialoux G, 2011. Consequences of HIV infection on malaria and therapeutic implications: a systematic review. Lancet Infect Dis 11: 541-556.

92. Rutto EK et al., 2015. Effects of HIV-1 infection on malaria parasitemia in milo sub-location, western Kenya. BMC Res Notes 8: $1-5$.

93. Hernán M, Robins JM, 1999. Letter to the editor: assessing the sensitivity of regression results to unmeasured confounders in observational studies. Biometrics 55: 1316-1317.

94. Lal AA, 2004. Interactions of multiple infectious agents in malariaendemic areas: concurrent HIV/AID and malaria. Knobler S, O'Connor S, Lemon S, eds. The Infectious Etiology of Chronic Diseases: Defining the Relationship, Enhancing the Research, and Mitigating the Effects: Workshop Summary. Washington, DC: National Academies Press, e473-e480. Available at: https:// www.ncbi.nlm.nih.gov/books/NBK83694/. Accessed March 1, 2019.

95. Audu RA et al., 2005. Possible impact of co-infections of tuberculosis and malaria on the CD4+ cell counts of HIV patients in Nigeria. Ann Afr Med 4: 10-13.

96. Wiwanitkit V, 2006. Co-infection between tuberculosis and malaria: a consideration on interaction of molecules and pathogenesis. J Vector Borne Dis 43: 195-197. 